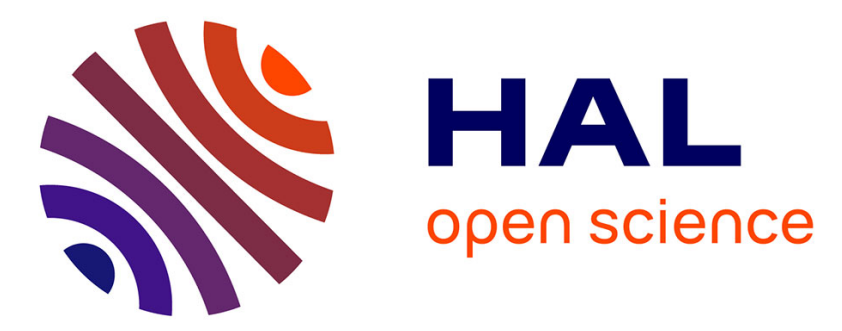

\title{
Measurement and modelling of the reflection coefficient of an Acoustic Black Hole termination
}

\author{
Vivien Denis, François Gautier, Adrien Pelat, J Poittevin
}

\section{To cite this version:}

Vivien Denis, François Gautier, Adrien Pelat, J Poittevin. Measurement and modelling of the reflection coefficient of an Acoustic Black Hole termination. Journal of Sound and Vibration, 2015, 349, pp.67-79. 10.1016/j.jsv.2015.03.043 . hal-01288278

\section{HAL Id: hal-01288278 https://hal.science/hal-01288278}

Submitted on 14 Mar 2016

HAL is a multi-disciplinary open access archive for the deposit and dissemination of scientific research documents, whether they are published or not. The documents may come from teaching and research institutions in France or abroad, or from public or private research centers.
L'archive ouverte pluridisciplinaire HAL, est destinée au dépôt et à la diffusion de documents scientifiques de niveau recherche, publiés ou non, émanant des établissements d'enseignement et de recherche français ou étrangers, des laboratoires publics ou privés. 


\title{
Measurement and modelling of the reflection coefficient of an Acoustic Black Hole termination
}

\author{
V. Denis ${ }^{1}$, F. Gautier ${ }^{1}$, A. Pelat ${ }^{1}$, J. Poittevin ${ }^{1}$ \\ Laboratoire d'Acoustique de l'Université du Maine, UMR CNRS 6613, Avenue Olivier \\ Messian, 72085 Le Mans, Cedex 09
}

\begin{abstract}
The flexural waves propagating in a beam can be efficiently absorbed if one extremity is tapered with a power law profile and covered by a thin layer of additional damping material. Such a termination induces the so-called "acoustic black hole effect" (ABH): if the thickness decreases locally, flexural waves slow down and the amplitude of the displacement field increases, leading to efficient energy dissipation if an absorbing layer is placed where the thickness is minimum. This paper presents a specific study of the reflection coefficient of $\mathrm{ABH}$ beam terminations. A Kundt-like measurement method of the reflection coefficient of a beam termination is proposed. The method is validated using theoretical results in the case of a beam free end. Results for several ABH extremities show a clear decrease of the modulus of the reflection coefficient $R$. The phase of $R$ due to the decreasing thickness profile is also investigated and is interpreted by defining a correction length for the tapered termination. These experimental results are compared with several models: geometrical acoustics based model, beam waveguide model
\end{abstract}

Email address: vivien.denis@univ-lemans.fr (V. Denis) 
and plate model.

Keywords:

reflection coefficient, wave method, flexural vibration, damping, acoustic black hole, inhomogeneous structural waveguide, correction length

\section{Introduction}

The reduction of unwanted vibrations is important for structural security, stability and comfort in many industrial applications. A classical vibration damping method consists in using surface damping treatments. Indeed, these

methods have proven to be very efficient $[1,2]$, although they often result in an increased mass of the treated structure. This may be unacceptable for ecological and economical reasons in the transport industry for example. It is then of a great interest to develop lightweight vibration damping methods.

It is well known that the sharpness of the resonances of a structure depends on both the damping properties of the constitutive material and the boundary conditions [3]. Therefore, for a given beam structure made of a particular material, a reduction of the resonant behaviour can be obtained by attenuating bending wave reflections at the edges. In this matter, a method using a graded impedance interface at the edges of a beam is proposed by Vemula et al. [3], and consists in assembling several pieces of beams made with different materials. The energy dissipation within the composite material at the free end, coupled with relatively large amplitude vibrations caused by the impedance gradation, results in a smaller reflectivity. The so-called Acoustic Black Hole (ABH) effect can also be used to reduce the reflection 
coefficient of a beam free end $[4,5,6]$. The $\mathrm{ABH}$ effect can be obtained from the bending wave properties in a beam of decreasing thickness: assuming geometrical acoustics hypothesis, Mironov [7] shows that in the neighbourhood of a beam edge whose thickness decreases smoothly to zero, flexural waves slow down and stop without being reflected. The condition of sufficient smoothness can be fulfilled by a power-law thickness profile $h(x)$ in the form:

$$
h(x)=\epsilon x^{m},
$$

where $x$ is the spatial coordinate and $m \geq 2$. If the thickness is strictly zero at the edge [7], it can be shown that the time taken by a wave to reach the edge becomes infinite. For a practical structure with a finite thickness, the reflection coefficient tends to zero with the decrease of the residual thickness at the tip of the tapered profile. It is however shown that manufacturing processes are such that this residual thickness can never be small enough for the effect to be attractive.

35

Krylov et al. $[4,5]$ show, also in the framework of geometrical acoustics (i.e. ignoring attenuating flexural waves in the medium), that covering the tapered profile by a thin damping layer compensates the finite thickness at the edge of experimental structures. The obtained analytical reflection coefficient has drastically reduced values compared to a free uniform extremity. In the framework of Euler-Bernoulli beam modelling, a wave-based approach that takes into account the effect of attenuating waves has been proposed by Georgiev et al. [6]. From a wave-vector formulation, a Riccati equation is numerically solved to compute a mechanical impedance matrix along the 
tapered beam and to derive a reflection matrix. Some practical rules for determining the optimal geometrical and material properties of the damping layer are found using this model [8]. Further investigations from Denis et al. [9] show that a two-dimensional behaviour has to be taken into account to model the tapered zone: the small thickness of the extremity induces a 50 local plate behaviour in a beam with an $\mathrm{ABH}$ extremity, and local transverse eigenmodes can be found in the beam structure. Note that two-dimensional $\mathrm{ABH}$ have also been described in the literature: circular $\mathrm{ABH}$ used as plate vibration damper has been firstly proposed by Gautier et al. [10] and studied both experimentally $[10,11,12]$ and theoretically $[13,14,15]$.

55

A claim of the previous studies is that the $\mathrm{ABH}$ effect is a way to obtain a quasi-anechoic termination; yet, most of the experimental studies observe frequency responses and not an actual reflection coefficient, which can be considered as a more direct indicator. Although this method has been developed for $\mathrm{ABH}$ termination, it remains applicable to any arbitrary beam end. This article focuses on the measurement of the reflection coefficient for flexural waves in a beam which carries an $\mathrm{ABH}$ on its extremity. First, the experimental setup is described and the measurement method is detailed in Sec. 2. In Sec. 3, the experimental results for the reflection coefficient of a uniform beam and several ABH beams are shown and commented. Sec. 4 presents different models of the literature and compares them with the experimental results. A conclusion is given in Sec. 5 . 


\section{Measurement method}

Reflection in beams has been thoroughly studied $[16,17]$. Several methods

70 allow the estimation of reflection properties of beam extremities. Linjama et al. $[18,19]$ propose to measure the reflection of bending waves using a structural intensity technique. Vemula et al. [3] estimate the reflected energy of bending waves in a graded impedance interface using the half power bandwidth of the resonance peaks of the transfer function. Carniel et al. [20] and Piaud et al. [21] both propose expressions for the far field reflection coefficient in a beam. Moulet et al. $[22,23]$ use a Kundt tube-like technique in order to estimate the scattering properties of a junction between two beams from the measurements of velocities along the beam. A method inspired by $[22,23]$ is used in the current paper.

\subsection{Experimental setup}

\subsubsection{Setup}

The estimation of the reflection coefficient is based on mobility transfer functions measurements. In order to avoid static deformation due to gravity, the beam to be tested is hanged vertically (see Fig. 1a and b). The extremity whose reflection coefficient is estimated is oriented towards the bottom. An impedance head (B\&K type 8001) and a shaker (B\&K type 4810) are glued on the beam. The shaker is suspended with three elastic wires. The velocity at each point of the mesh is measured using a laser vibrometer (Polytec OFV 303). The impedance head, shaker and vibrometer are linked to a LMS ac-

90 quisition system (Scadas Mobile 4 channels, Test.Lab Rev 13) which acquires force and acceleration signals at the excitation point and velocity signal at 


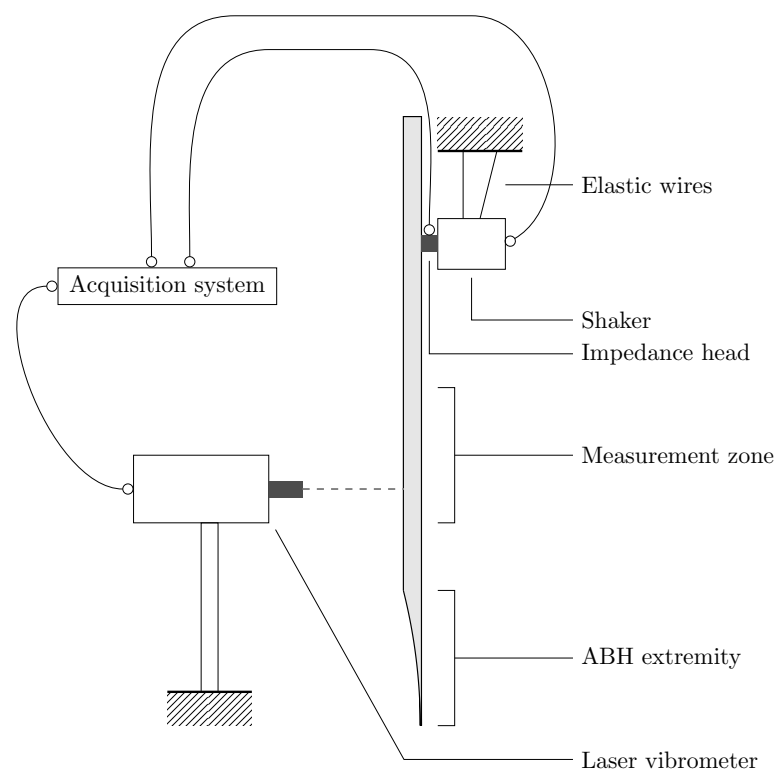

(a)

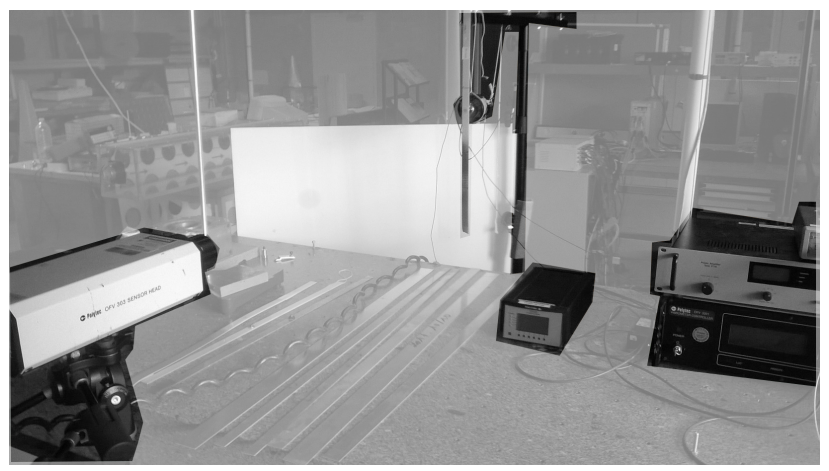

(b)

Figure 1: (a) Scheme of the experiment. (b) Photo of the experimental setup. 
measurement points.

\subsubsection{Samples}

95

100

Two constitutive materials are used for the beams: aluminium and polymer (Vero White Plus FullCure 835). For each material, two beams are tested: a uniform beam (i.e. constant cross section) used as a reference for the estimation method, and a beam with an $\mathrm{ABH}$ termination, whose geometry is described on Fig. 2. A layer of damping material made with viscoelastic tape is stuck on the flat side of the tapered profile. The dimensions and material characteristics (Young modulus, mass density, loss factor and Poisson coefficient) of the samples are given in Tab. 1.

The ABH termination for the aluminium beam is manufactured using a conventional machining process, allowing to obtain a minimum thickness at the extremity of about $20 \mu \mathrm{m}$. The process requires high precision work and often generates irregularities and tearing at the tip of the profile. The loss factor for aluminium has an arbitrarily chosen low value reflecting a very lightly damped beam and allowing to obtain convincing results. The polymer beam is manufactured using UV polymerisation, which is a rapid prototyping process. This manufacturing process allows a thinner minimum thickness of about $16 \mu \mathrm{m}$ to be obtained and enables a better control on the profile but restricts the choice of the material. For this material, a standard measurement of the complex Young modulus versus frequency has been done.

The results of the Dynamical Mechanical Analysis (DMA)[24] between 0.4 and $40 \mathrm{~Hz}$ have been extrapolated on the $0-12 \mathrm{kHz}$ range using the Williams- 
(a)
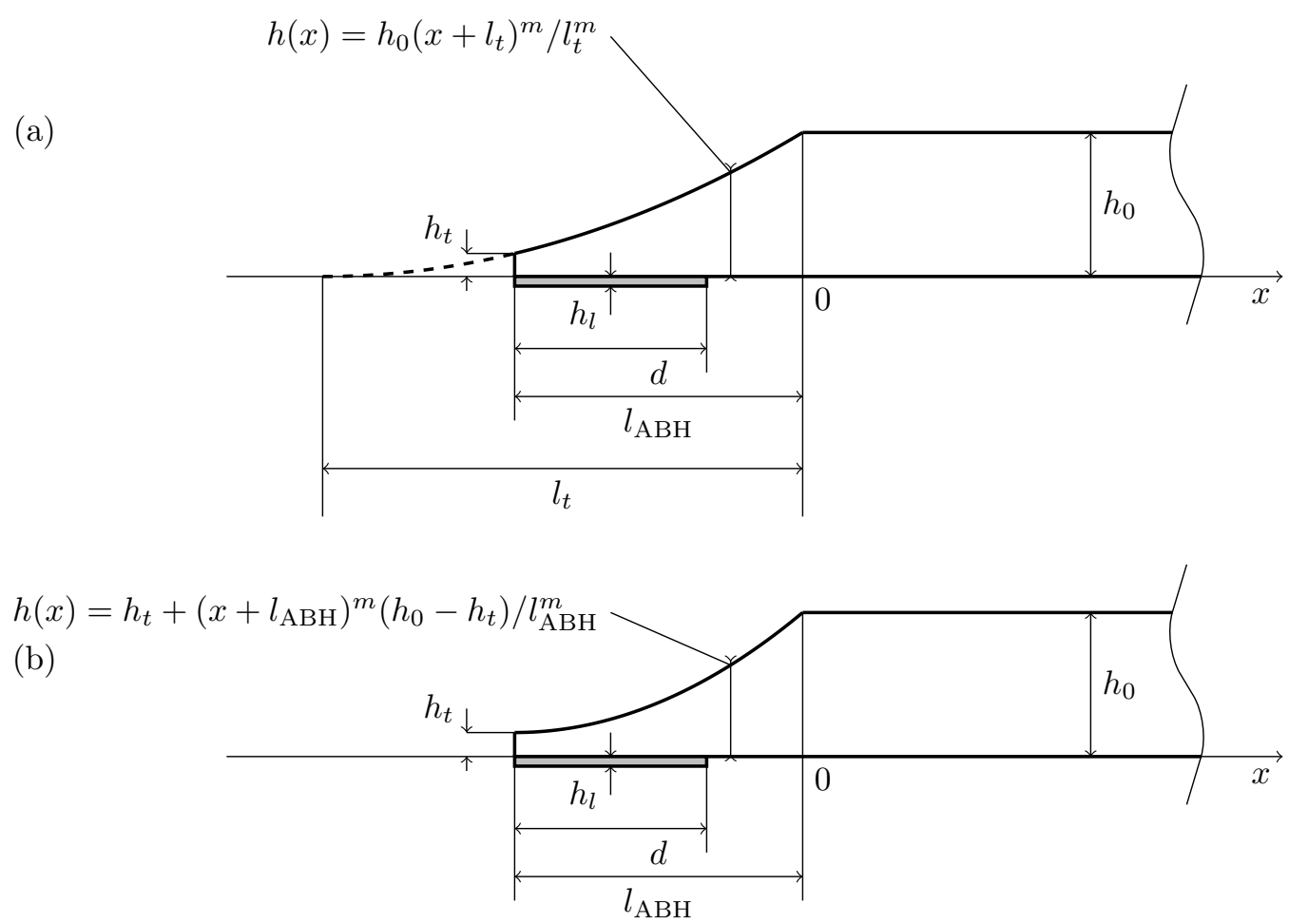

Figure 2: Scheme of the ABH profile at beam extremity for (a) aluminium and (b) polymer cases.

Landel-Ferry model [25]. They provide the indicative values given in Tab. 1. Variable complex Young modulus from the DMA results has been actually used in the following and is plotted on Fig. 3.

\subsubsection{Measurement conditions}

Transfer mobility measurements are performed at 21 abscissas $5 \mathrm{~mm}$ apart from each other. In the case of the aluminium ABH beam, the closest abscissa is located $0.1 \mathrm{~m}$ way from the tapered region. Let $A_{0}$ be the point on the neutral axis of the beam at absissa $x_{i}$ (see Fig. 4a). Let $A_{l}$ and $A_{r}$ be two 

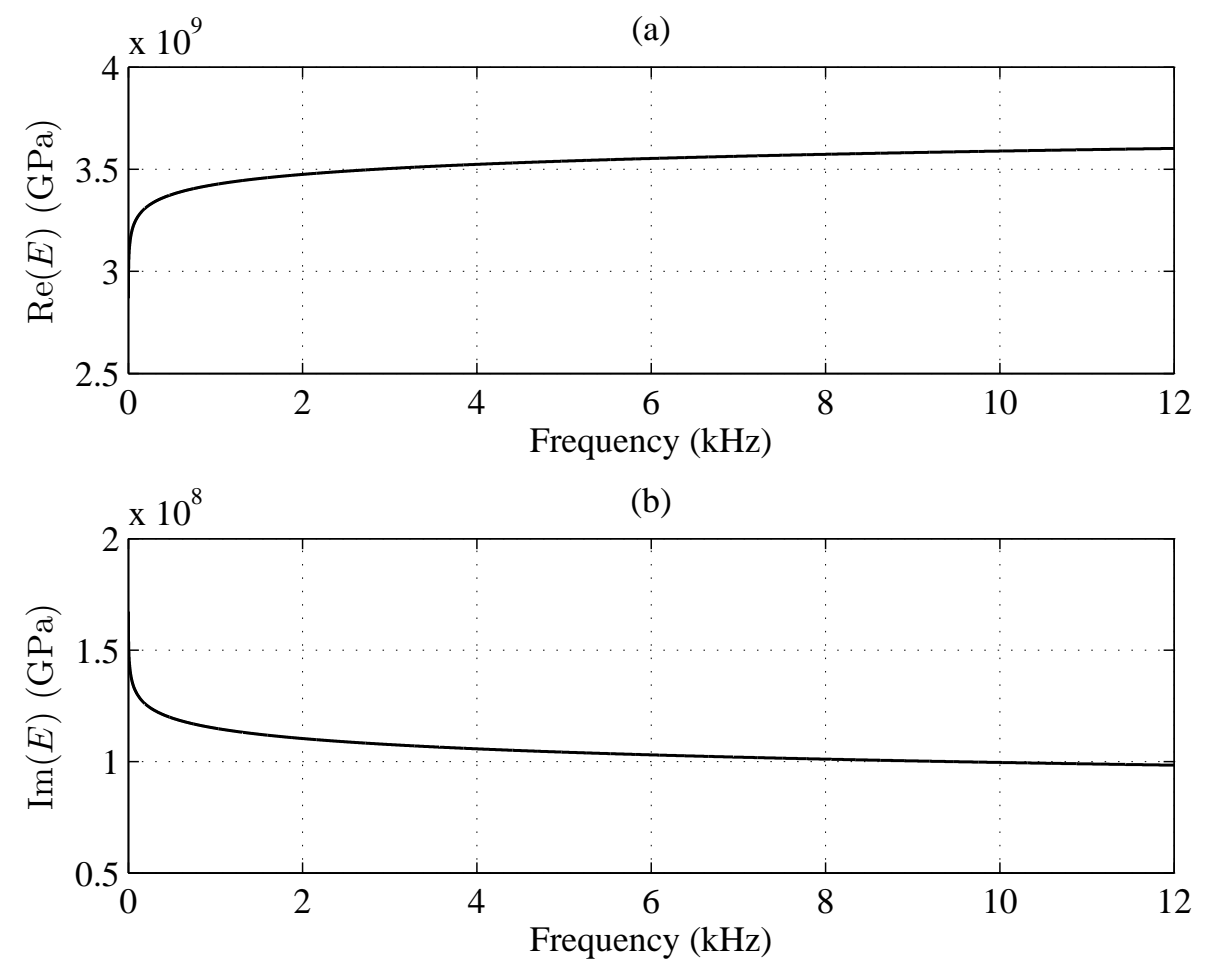

Figure 3: Complex variations of (a) the real part and (b) the imaginary part of the Young modulus $E$ of the polymer material. 
Table 1: Geometrical and material characteristics of the sample beams and damping layer.

\begin{tabular}{|c|c|}
\hline Geometrical characteristics & Characteristics of material \\
\hline \multicolumn{2}{|c|}{ Aluminium beam } \\
\hline $\begin{array}{l}L=0.8 \mathrm{~m}, b=0.02 \mathrm{~m}, \\
l_{\mathrm{ABH}}=0.06 \mathrm{~m}, l_{t}=0.07 \mathrm{~m}, \\
h_{0}=1.5 \mathrm{~mm}, m=2 .\end{array}$ & $\begin{array}{l}E_{1}=70 \mathrm{GPa}, \eta_{1}=0.1 \%, \\
\rho_{1}=2700 \mathrm{~kg} \cdot \mathrm{m}^{-3}, \nu=0.3 .\end{array}$ \\
\hline \multicolumn{2}{|c|}{ Polymer beam } \\
\hline $\begin{array}{l}L=0.6 \mathrm{~m}, b=0.02 \mathrm{~m}, \\
l_{\mathrm{ABH}}=0.06 \mathrm{~m}, h_{t}=0.16 \mathrm{~mm} \\
h_{0}=1.5 \mathrm{~mm}, m=2 .\end{array}$ & $\begin{array}{l}E_{1} \simeq 3.5 \mathrm{GPa}, \eta_{1} \simeq 2.8 \% \\
\rho_{1}=1175 \mathrm{~kg} \cdot \mathrm{m}^{-3}, \nu=0.3\end{array}$ \\
\hline \multicolumn{2}{|c|}{ Damping layer } \\
\hline $\begin{array}{l}h_{l}=0.1 \mathrm{~mm}, \\
d=0.05 \mathrm{~m} .\end{array}$ & $\begin{array}{l}E_{2}=7 \mathrm{GPa}, \eta_{2}=40 \%, \\
\rho_{2}=1000 \mathrm{~kg} \cdot \mathrm{m}^{-3} .\end{array}$ \\
\hline
\end{tabular}

points at the right and left hand side of the neutral axis, with distances $a_{l}=A_{l} A_{0}$ and $a_{r}=A_{r} A_{0}$, respectively. Let $E$ and $F(E)$ be the excitation point at abscissa $x_{f}$ and the force applied in $E$, respectively. The studied reflection coefficient concerns only flexural waves. The measured velocity $\dot{W}_{i}$ at a given abscissa $x_{i}$ can be written as

$$
\dot{W}_{i}=\dot{W}_{i f}+\dot{W}_{i t}
$$

where $\dot{W}_{i f}$ and $\dot{W}_{i t}$ correspond to pure beam flexural motion and beam torsional motion, respectively. Only the flexural contribution should be estimated. The mobility on the neutral axis, where there is no torsional contribution, is defined as

$$
Y\left(A_{0}, E\right)=\frac{\dot{W}_{i f}}{F(E)} .
$$


It can be shown that

$$
Y\left(A_{0}, E\right)=\frac{a_{l} Y\left(A_{l}, E\right)+a_{r} Y\left(A_{r}, E\right)}{a_{l}+a_{r}}=\frac{\alpha Y\left(A_{l}, E\right)+Y\left(A_{r}, E\right)}{1+\alpha},
$$

where $\alpha=a_{l} / a_{r}$. The $\alpha$ coefficient is adjusted in order to minimize the amplitude of the first torsional peak, allowing to find $Y\left(A_{0}, E\right)$ from the measurements of $Y\left(A_{l}, E\right)$ and $Y\left(A_{r}, E\right)$. The effect of torsion on the wave field is therefore efficiently reduced on a wide frequency range for each abscissa. Fig. 4b shows the mobility transfer function between the excitation and a random measurement abscissa for the uniform aluminium beam at left and right hand sides of the centreline (full and dashed gray lines). The transfer function on the neutral axis (thick black line) is also plotted and shows that the first torsional peak at $197 \mathrm{~Hz}$ vanishes. Mobilities of the uniform and $\mathrm{ABH}$ beams made of aluminium are plotted on Fig. 4c; the torsional peaks are minimized and the smoothing effect of the $\mathrm{ABH}$ treatment can be noticed.

The quality of the transfer function measurements is estimated using ordinary coherence function. It is easily shown that excitation level has to be large enough to get coherence function close to 1 . To make sure that this condition is valid over a wide frequency range, the whole useful band 0-12 $\mathrm{kHz}$ is split in two bands: $0-2 \mathrm{kHz}$ and $2-12 \mathrm{kHz}$ for which two different levels are applied for the swept sine excitation. Hence, for each couple $\left(E, A_{0}\right)$, two sets of measurements are performed in order to compute the mobility. 


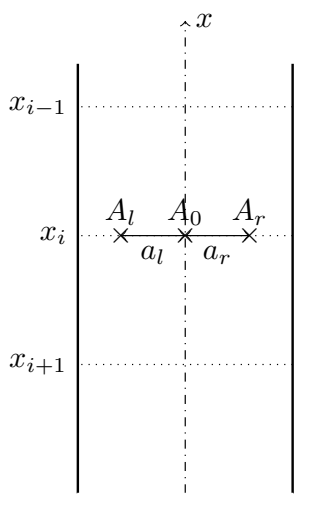

(a)

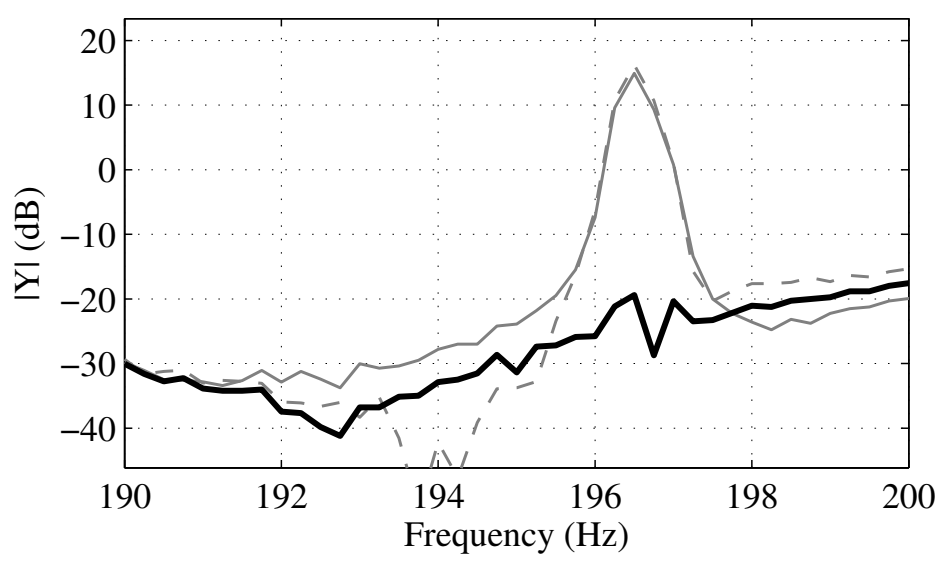

(b)

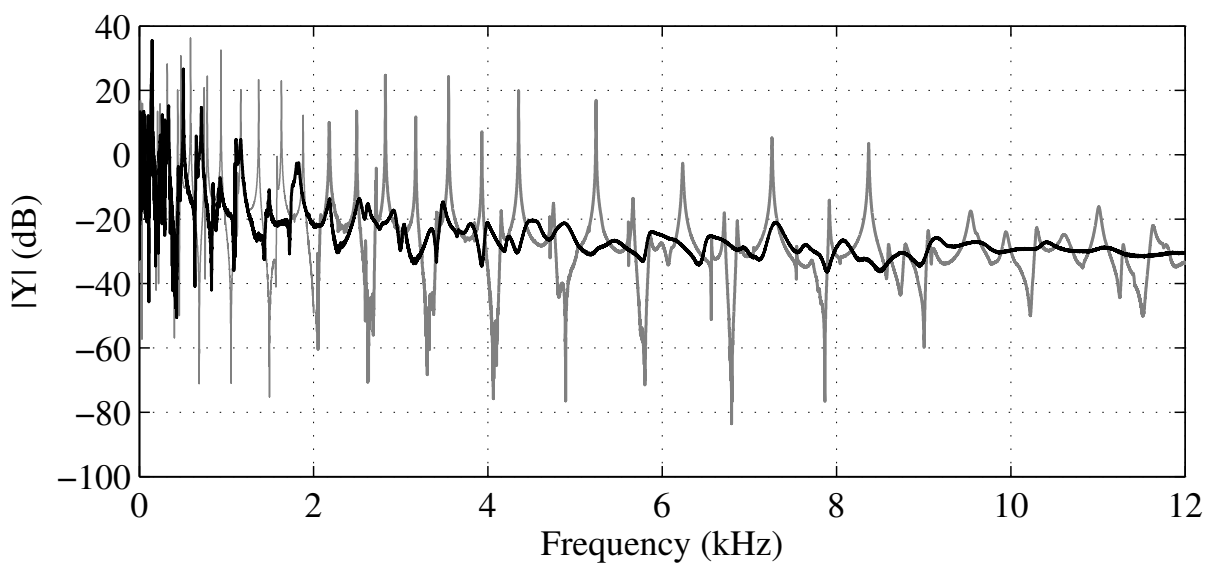

(c)

Figure 4: (a) Left, neutral axis and right measurement points $A_{l}, A_{0}$ and $A_{r}$ at abscissa $x_{i}$. (b) Transfer mobility measurement $Y\left(A_{i}, E\right)$ (full gray line), $Y\left(A_{r}, E\right)$ (dashed gray line) and $Y\left(A_{0}, E\right)$ (thick full black line) on the uniform aluminium beam (dB, ref. $\left.1 \mathrm{~m} . \mathrm{s}^{-1} \cdot \mathrm{N}^{-1}\right)$. Force is applied at $x_{f}=0.58 \mathrm{~m}$, velocity is measured at $x_{i}=0.3$ m. (c) Transfer mobility measurement $Y\left(A_{0}, E\right)$ for uniform (gray line) and ABH (black line) beams made of aluminium $\left(\mathrm{dB}\right.$, ref. $\left.1 \mathrm{~m} \cdot \mathrm{s}^{-1} \cdot \mathrm{N}^{-1}\right)$. Force is applied at $x_{f}=0.58 \mathrm{~m}$, velocity is measured at $x_{i}=0.3 \mathrm{~m}$. 
155

\subsection{Methodology for the estimation of the reflection coefficient}

\subsubsection{Wave field solution for beams}

Let us consider a beam of constant cross section whose extremity $x=0$ bears an $\mathrm{ABH}$ profile seen as an unknown boundary condition (see Fig. 5). Under the Euler-Bernoulli assumptions, the equation of harmonic flexural motion $W(x, \omega)$ of such a beam in the absence of excitation, and assuming a harmonic motion with time dependency $e^{-j \omega t}$, is [17]

$$
E^{*} I \frac{\partial^{4} W(x, \omega)}{\partial x^{4}}-\rho S \omega^{2} W(x, \omega)=0 \quad \forall x \in[0, L],
$$

where $\omega$ is the angular frequency, $E^{*}=E(1+\mathrm{j} \eta)$ is the complex Young modulus (with $E$ the storage modulus and $\eta$ the loss factor), $I$ is the moment of inertia of the cross-section, $\rho$ is the mass density, $S$ is the cross-section area and $L$ is the length of the uniform part of the beam.

The general solution for the displacement can be written as the sum of four waves [17]:

$W(x, \omega)=A(\omega) e^{-j k_{f} x}+B(\omega) e^{+j k_{f} x}+C(\omega) e^{-k_{f} x}+D(\omega) e^{+k_{f} x} \quad \forall x \in[0, L]$

where the flexural wavenumber $k_{f}$ is such that

$$
k_{f}^{4}=\frac{\rho S}{E^{*} I} \omega^{2}
$$

170

The scalar coefficients $A, B, C$ and $D$ represent the magnitude of backward and forward propagating and attenuating waves, respectively (see Fig. 5). 
At the ABH extremity or the free extremity $(x=0)$, the boundary conditions associated to the equation of motion (5) can be written as a reflection

175 matrix $\mathbf{R}$ such that

$$
\left[\begin{array}{l}
A \\
C
\end{array}\right]=\mathbf{R}\left[\begin{array}{l}
B \\
D
\end{array}\right],
$$

where

$$
\mathbf{R}=\left[\begin{array}{ll}
R_{p p} & R_{a p} \\
R_{p a} & R_{a a}
\end{array}\right]
$$

with $R_{i j}$ the reflection coefficient between incident wave $i$ and reflected wave $j ; i$ and $j$ symbolize $p$ and $a$ standing for propagating and attenuating respectively.

We are interested in measuring terms of $\mathbf{R}$. The boundary condition at $x=\mathrm{L}$ has to be defined for the experiment but do not play any role in the measurements of $\mathbf{R}$. In practice, free boundary conditions are supposed at $x=\mathrm{L}$.

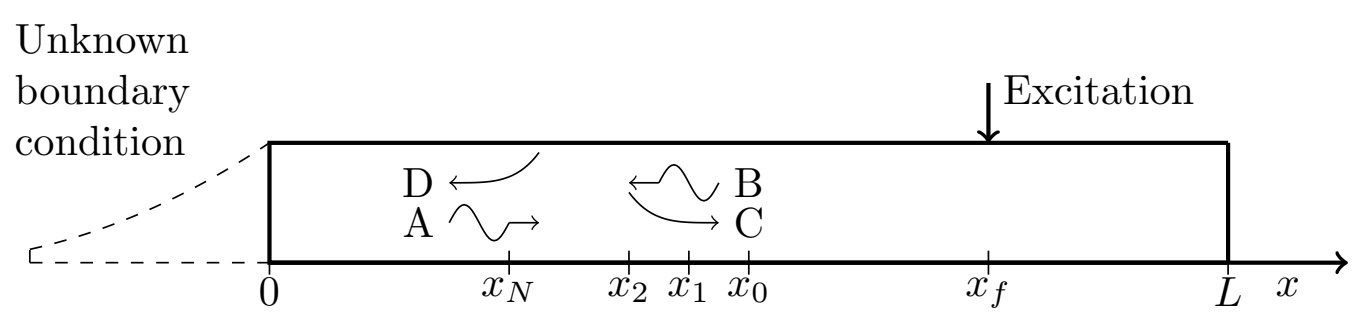

Figure 5: Scheme of the beam showing the excitation point $x_{f}$, the $N+1$ measurement points $x_{0}$ to $x_{N}$, the attenuating and propagating waves and the boundary condition at $x=0$. 
185

\subsubsection{Far field assumption}

Far from the excitation point and from the boundaries, attenuating waves vanish and the wave field can be well approximated as the sum of two propagating waves only:

$$
W(x, \omega)=A(\omega) e^{-j k_{f} x}+B(\omega) e^{+j k_{f} x} .
$$

The boundary condition can then be described with a far field reflection 190 coefficient $R$ that corresponds to $R_{p p}$ in Eq. (9). It is assumed that the far field assumption is valid at a given frequency when the attenuating waves coming from an excitation point or a boundary condition lose $90 \%$ of their magnitude over the propagation length $l$. Therefore, there should be

$$
\left\{\begin{array}{l}
x_{N}>l, \\
x_{f}-x_{0}>l,
\end{array}\right.
$$

where the distance $l$ satisfies [22]

$$
e^{-k_{f} l}=0.1
$$

In the experiment described in Sec. 2.1, the closest measurement point is at $l=0.1 \mathrm{~m}$ of the tapered zone of the aluminium $\mathrm{ABH}$ beam, so that the far field assumption is theoretically valid above $200 \mathrm{~Hz}$.

\subsubsection{Estimation of the wave and reflection coefficients}

Let us consider $N+1$ points along the beam satisfying the far field assumption (see Fig. 5). The displacement measured for all abscissas $x_{i}(i \in[0, N])$ 
can be related to the coefficients $A$ and $B$ through the matrix equation

$$
\left[\begin{array}{c}
W\left(x_{0}, \omega\right) \\
W\left(x_{1}, \omega\right) \\
W\left(x_{2}, \omega\right) \\
\ldots \\
W\left(x_{N}, \omega\right)
\end{array}\right]=\underbrace{\left[\begin{array}{cc}
e^{-j k_{f} x_{0}} & e^{j k_{f} x_{0}} \\
e^{-j k_{f} x_{1}} & e^{j k_{f} x_{1}} \\
e^{-j k_{f} x_{2}} & e^{j k_{f} x_{2}} \\
\ldots & \ldots \\
e^{-j k_{f} x_{N}} & e^{j k_{f} x_{N}}
\end{array}\right]}_{\mathbf{M}}\left[\begin{array}{c}
A(\omega) \\
B(\omega)
\end{array}\right],
$$

with $\mathbf{M}$ a $(N \times 2)$ matrix. A least-squares estimation of the $[A, B]^{T}$ vector can be effectively obtained with

$$
\left[\begin{array}{c}
A(\omega) \\
B(\omega)
\end{array}\right]=\mathbf{M}^{\dagger}\left[\begin{array}{c}
W\left(x_{0}, \omega\right) \\
W\left(x_{1}, \omega\right) \\
W\left(x_{2}, \omega\right) \\
\ldots \\
W\left(x_{N}, \omega\right)
\end{array}\right],
$$

where $\mathbf{M}^{\dagger}=\left(\mathbf{M}^{*} \mathbf{M}\right)^{-1} \mathbf{M}^{*}$ is the Moore-Penrose generalized inverse [26] of $\mathbf{M}$ and $\mathbf{M}^{*}$ is the conjugate transpose of $\mathbf{M}$.

From this estimation of the wave coefficients, the far field reflection coefficient of the extremity can be derived:

$$
R(\omega)=\frac{A(\omega)}{B(\omega)} .
$$




\subsection{Validation on the uniform beam}

It can be shown that the reflection coefficient for a free end is given by $[17]$

$$
R=\frac{A}{B}=-\mathrm{j} .
$$

This result is used to validate the proposed measurement method. Fig. 6 presents the reflection coefficient of the free end at $x=0$ measured on aluminium (full black line), estimated with the method described in Sec. 2. It is checked that the theoretical result is experimentally obtained: modulus and phase are constant with respect to frequency and equal to 1 and $-\frac{\pi}{2}$, respectively. It is remarkable to see that the experimental result of Fig. 6

Fig. 6 also shows the reflection of the free end in the polymer case with the assumed material characteristics of Tab. 1 (full light gray line). The unexpected phase increase suggests an incorrect knowledge of the wavenumber. Indeed, modulus and phase of the reflection coefficient are related to the imaginary and real parts of the wavenumber, respectively. The wavenumcoefficient with the free end theoretical result, in modulus and phase. The fit made on the characteristics of the polymer material consists in increasing by $2 \%$ the real part of the wavenumber calculated with characteristics of 
Tab. 1, in order to obtain the theoretical reflection coefficient of a free end ( $E_{1} \simeq 3.8 \mathrm{GPa}$ ). Modulus and phase of $R$ with fitted wavenumber (see Fig. 6, dashed dark gray line) are constant and show that the estimation method relies on a precise knowledge of the wavenumber.

However, it can be noticed that the reflection coefficient for the polymer beam behaves poorly after $7 \mathrm{kHz}$. This can be expected since the structure cannot be considered anymore as a beam above this frequency and is likely to display a two-dimensional plates behaviour, because the wavelength $\left(\lambda_{f=7 \mathrm{kHz}}^{\text {Polymer }} \simeq 0.026 \mathrm{~m}\right)$ neighbours the width of the cross-section, that is

$$
\lambda_{f=7 \mathrm{kHz}}^{\text {Polymer }} \simeq 1.3 b
$$

Consequently, the estimation based on one-dimensional wave decomposition does not make sense above $7 \mathrm{kHz}$ for the polymer beams. This issue does not appear with the aluminium beam in which the wavelength is much larger than the beam width in the $0-12 \mathrm{kHz}$ range $\left(\lambda_{f=12 \mathrm{kHz}}^{\text {Aluminium }} \simeq 1.7 \mathrm{~b} \simeq 0.034 \mathrm{~m}\right)$. It is also noted that in the two beams, the wavelength is much larger than the beam thickness, justifying the use of the classical Euler-Bernoulli beam theory.

Small singularities appears on the curves, especially for the aluminium beam. This is due to a drop of coherence at anti-resonances. A more damped structure such as the polymer beam shows less coherence drops and thus, a 255 smoother curve for the reflection coefficient. 
(a)

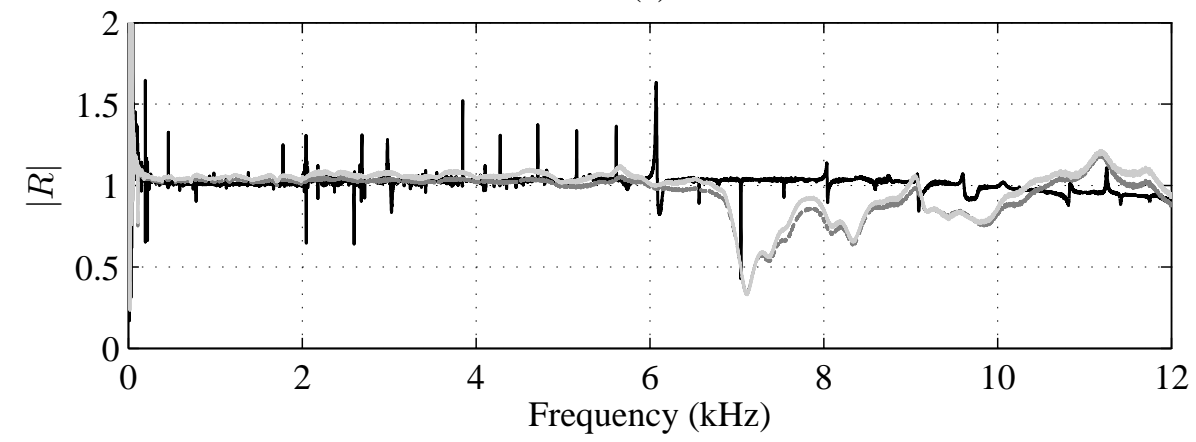

(b)

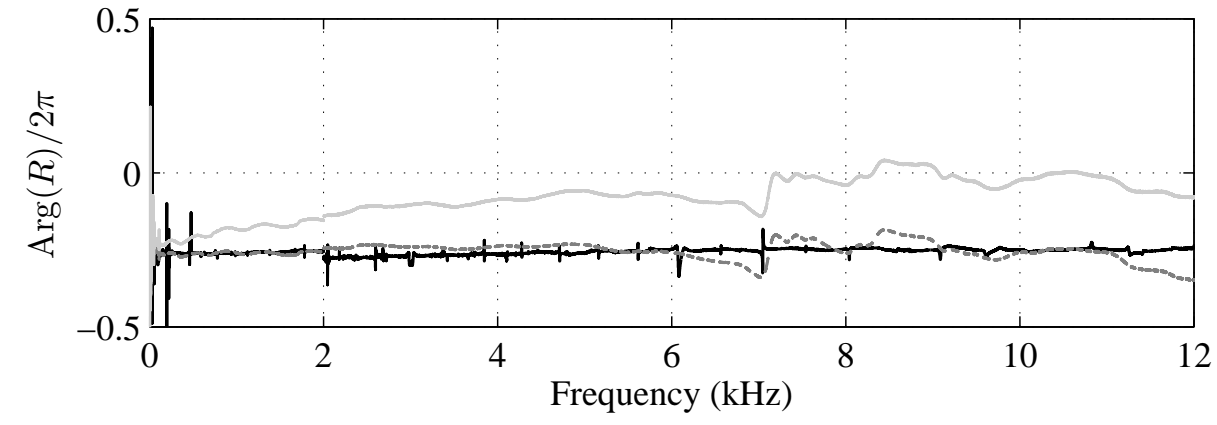

Figure 6: Estimated reflection coefficient $R$ for the free end of uniform beams made of aluminium (full black line), polymer with fitted wavenumber (dashed dark gray line) and polymer with non-fitted wavenumber (full light gray line), in (a) modulus and (b) phase. 


\subsection{Results on $A B H$ beams}

\subsubsection{Typical results}

Fig. 7 shows the estimated reflection coefficient for the ABH extremity

260 dark gray line) beams. Compared to the free end, it is very clear that the modulus of $R$ decreases with increased frequency. Values in modulus can be as small as 0.1. Moreover, oscillations can be observed on the modulus. The phase of the reflection coefficient displays a significant additional roll down 265

Qualitative variations of $|R|$ are similar for the aluminium and polymer cases. The modulus of the reflection coefficient is smaller for the polymer beam, showing that the $\mathrm{ABH}$ is more efficient in this material. The phase also rolls down faster in the polymer case below $7 \mathrm{kHz}$.

\subsubsection{Phase of the reflection coefficient and equivalent length of the tapered profile}

Special attention is paid to the phase of the reflection coefficient. Instead of estimating the reflection coefficient at $x=0$ (see Fig. 6) for the uniform aluminium beam (named $R_{\text {Ref }}$ ), it is estimated at distance $l_{\mathrm{ABH}}$ (see Fig. 2) from the edge and plotted on Fig. 7 (full light gray line). It is compared with the phase the reflection coefficient of the aluminium $\mathrm{ABH}$ termination (named $R_{\mathrm{ABH}}$ ). The phase discrepancies between $R_{\text {Ref }}$ and $R_{\mathrm{ABH}}$ are studied in order to investigate the role of the $\mathrm{ABH}$ thickness profile. The phase of ${ }_{280} R_{\mathrm{ABH}}$ decreases more than the phase of $R_{\mathrm{Ref}}$, for the same actual termination length. It means that an incident wave spends more time in the tapered 
(a)

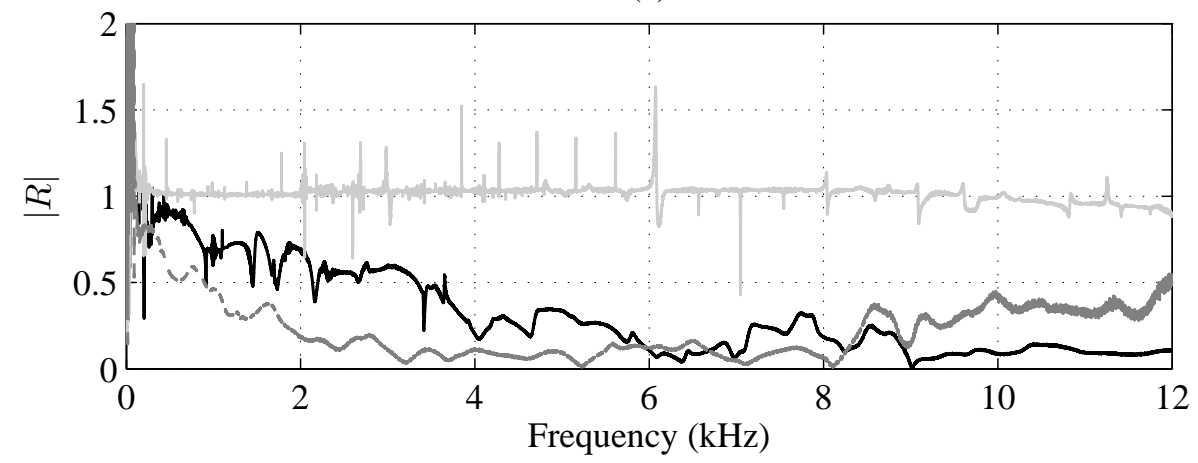

(b)

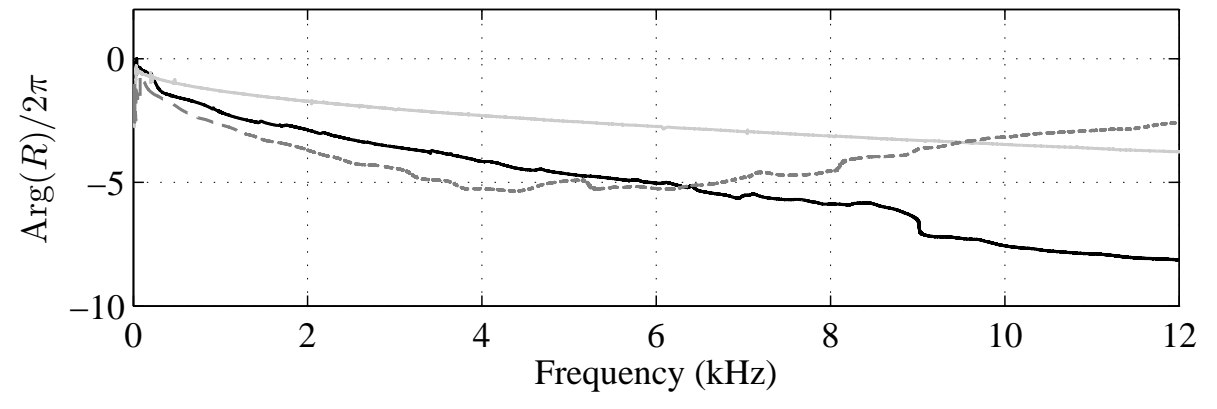

Figure 7: Estimated reflection coefficient $R$ for the termination of the aluminium (full black line) and polymer (dashed gray line) $\mathrm{ABH}$ beams and reflection coefficient for the reference beam estimated at distance $l_{A B H}$ of the free boundary condition (full light gray line), in (a) modulus and (b) phase. 
profile because it is slowed down. An alternative point of view is to consider a uniform profile of larger length instead of the tapered profile, in which the bending wave speed remains constant. This leads to define a correction length theoretically shown by Mironov [28] in the case of a retarding tube termination, constituting the acoustical analogy of the ABH termination for flexural 
waves studied in this paper.

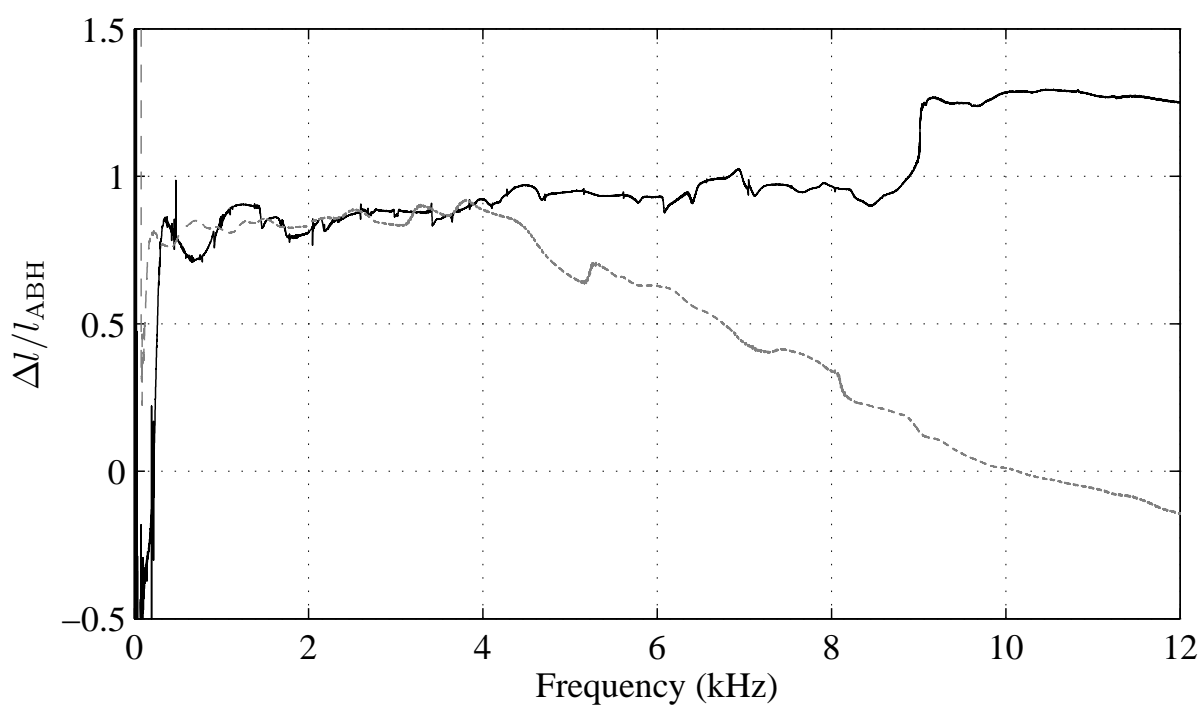

Figure 8: Measured dimensionless correction length $\Delta l / l_{\mathrm{ABH}}$ due to $\mathrm{ABH}$ termination for aluminium (full black line) and polymer (dashed gray line) ABH beams.

\section{Comparison with theoretical results}

Three models of the literature are used in this section in order to represent the reflection coefficient of an $\mathrm{ABH}$ beam extremity.

\subsection{Wave model based on geometrical acoustics assumptions} of a damping layer in the $\mathrm{ABH}$ framework. Considering geometrical acoustics assumptions, the reflection coefficient of the truncated wedge is calculated 
using the expression

$$
|R(0, \omega)|=\exp \left(-\int_{-l_{\mathrm{ABH}}}^{0} \operatorname{Im}(k(x, \omega)) d x\right),
$$

where $k(x, \omega)$ is the flexural wavenumber and $l_{\mathrm{ABH}}$ the length of truncated profile (see Fig. 2). The reflection coefficient is evaluated at $x=0$. Note that $[29,4]$ give only an information about the modulus of the reflection coefficient. In the proposed approach, attenuating waves are not taken into account. In [4], the author proposes two ways to take the damping layer into account in the expression of the wavenumber, depending on the relative thickness of the layer. We are interested here in the expression of the wavenumber $k$ when the layer is relatively thick compared to the plate (see Eq. (15-16) in [4]):

$$
\begin{aligned}
& k=\frac{12^{1 / 4} k_{p}^{1 / 2}\left(1+\rho_{2} h_{2}\right)^{1 / 4}}{h^{1 / 2}\left[1+e_{2} h_{2}\left(3+6 h_{2}+4 h_{2}^{2}\right)\right]^{1 / 4}} \\
& \cdot\left(1+\mathrm{j} \frac{1}{4} \frac{\eta_{1}+\eta_{2} e_{2} h_{2}\left(3+6 h_{2}+4 h_{2}^{2}\right)}{1+e_{2} h_{2}\left(3+6 h_{2}+4 h_{2}^{2}\right)}\right),
\end{aligned}
$$

where $h$ is the thickness of the plate alone, $\eta_{1}$ and $\eta_{2}$ are the loss factors of the plate and the layer, $e_{2}$ the ratio of the Young moduli of the layer and the beam, $h_{2}$ the ratio of the thicknesses of the layer and the beam, $\rho_{2}$ is the ratio of the mass density of the layer and the beam and $k_{p}=\omega \sqrt{\rho_{1}\left(1-\nu^{2}\right) / E_{1}}$ is the wavenumber of a plate wave and $\omega$ is the circular frequency. model for an $\mathrm{ABH}$ termination with parameters mentioned in Tab. 1. On this figure, $R$ is clearly monotonously decreasing. 


\subsection{Inhomogeneous wave model under plane wave assumption}

The ABH extremity can be considered as an heterogeneity in a structural

waveguide constituted by the beam. Several techniques exist in the literature to describe the vibratory field and compute a reflection coefficient: transfer matrix method, scattering matrix method, impedance matrix method, etc. $[16,30,31]$. If one considers the attenuating waves, impedance matrix method is the most efficient one. Georgiev et al. [6] propose a model that takes into account the attenuating part of the wave field to compute the reflection coefficient of an $\mathrm{ABH}$ beam extremity using an impedance matrix technique. Following the Euler-Bernoulli assumptions, the cross-section remains undeformed during the vibration. All points of the cross-section are thus in phase, which is the main feature of a plane wave. No transverse variations of the vibration field are considered. In [6], the state vector $\mathbf{X}$ describing the kinematics and the efforts along the beam respects the state equation

$$
\frac{\partial \mathbf{X}}{\partial x}=\mathbf{H X}
$$

where $\mathbf{H}$ is a matrix describing the governing equations of the Euler-Bernoulli model. Such formulation allows to develop the non linear Riccati equation for the impedance $\mathbf{Z}$ along the beam

$$
\frac{\partial \mathbf{Z}}{\partial x}=-\mathbf{Z H}_{1}-\mathbf{j} \omega \mathbf{Z} \mathbf{H}_{2} \mathbf{Z}+\frac{\mathbf{H}_{3}}{\mathbf{j} \omega}+\mathbf{H}_{4} \mathbf{Z}
$$

where $\mathbf{H}_{1}$ to $\mathbf{H}_{4}$ are the four submatrices of $\mathbf{H}$. A relation between the impedance matrix and a reflection matrix at a given point is then used. The damping layer effect is taken into account using equivalent material properties, according to [1]; the equivalent complex bending stiffness $E I^{*}(x)$ 
of the beam covered with a layer is expressed (see Eq. (B1) in [6])

$$
\begin{aligned}
E I^{*}=E_{1} I_{1}\left[\left(1+\mathrm{j} \eta_{1}\right)+e_{2}\right. & h_{2}^{3}\left(1+\mathrm{j} \eta_{2}\right) \\
& \left.+\frac{3\left(1+h_{2}\right)^{2} e_{2} h_{2}\left(1-\eta_{1} \eta_{2}+\mathrm{j}\left(\eta_{1}+\eta_{2}\right)\right)}{1+e_{2} h_{2}\left(1+\mathrm{j} \eta_{2}\right)}\right],
\end{aligned}
$$

where $E_{1} I_{1}$ is the bending stiffness of the beam alone, $\eta_{1}$ and $\eta_{2}$ are the loss factors of the plate and the layer, $e_{2}$ the ratio of the Young moduli of the layer and the beam and $h_{2}$ the ratio of the thicknesses of the layer and the beam. Note that Eq. (24) yields a wavenumber similar to Eq. (21).

The Ricatti equation (23) is solved numerically using a fourth-order RungeKutta scheme. As it is seen on Fig. 9 (dashed line), this model provides a result that differs from the model of [4] for the reflection coefficient. The geometrical model result is monotonously decreasing, without any oscillations. However, using the same parameters the same order of magnitude is obtained on the reflection coefficient. It can be assumed that the oscillations come from reflections throughout the $\mathrm{ABH}$ profile, suggesting that the sufficient smoothness condition [7] is not applicable in the studied frequency range for this $\mathrm{ABH}$ geometry. This subject has been studied by Feurtado et al. [32] who conclude that the sufficient smoothness condition is not verified at frequencies lower than $10 \mathrm{kHz}$ for $\mathrm{ABHs}$ with dimensions on the order of several centimeters. An information of phase is given by [6], while it is absent in the expression given by [4]. The phase rolls down and can give a hint about the travel time of a wave in the tapered region of the beam, as said in Sec. 3.2.2. 


\subsection{Inhomogeneous plate model}

In a previous work [9], the authors consider that the decreasing thickness in the $\mathrm{ABH}$ profile makes the beam locally behave like a plate even at low frequencies and investigate two-dimensional behaviour. A plate model with varying thickness along $x$ is used, in which the displacement $W(x, y)$ due to an harmonic excitation at $x_{f}$ of amplitude $F$ and angular frequency $\omega$ satisfies the following equation of motion:

$$
\begin{aligned}
\nabla^{2}\left(D(x) \nabla^{2} W(x, y)\right)-(1-\nu)\left(\frac{\partial^{2} D(x)}{\partial x^{2}} \frac{\partial^{2} W(x, y)}{\partial y^{2}}\right)- & \\
\rho(x) h(x) \omega^{2} W(x, y) & =F \delta\left(x-x_{f}\right),
\end{aligned}
$$

where $\rho(x)$ is the mass density, $h(x)$ is the thickness, $D(x)$ is the local complex bending stiffness and $\nu$ the Poisson coefficient. The damping layer effect

is taken into account in the same way than in Sec. 4.2 and allows to define the equivalent bending stiffness for the plate covered with damping layer. The problem is completely defined by using free boundary conditions on every edge of the plate.

This model is numerically solved from a finite difference scheme. This method uses a spatial mesh that is adapted to the thickness variation, in order to reduce the numerical dispersion. Thus a transformed grid is defined on which the wavelength remains constant spatially. Finally, the discretized problem leads to the matrix problem

$$
\left(\mathbf{K}-\omega^{2} \mathbf{M}\right) \mathbf{W}=\mathbf{F},
$$

where $\mathbf{K}$ and $\mathbf{M}$ are respectively the complex stiffness matrix and the mass matrix of the structure, $\mathbf{F}$ is the force vector and $\mathbf{W}$ is the displacement field. 
Using this numerical model, frequency responses are simulated and can be post-processed as described in Sec. 2. The result for the reflection coefficient is plotted on Fig. 9 (full line).

On Fig. 9, plane wave and plate models yield the same results when fed with the same parameters of Tab. 1. Actually, the phase of the reflection coefficient is slightly different; it can be attributed to a small numerical dispersion in the finite difference scheme of the plate model, leading to a difference between theoretical and actual wave speed in the structure. Considering that [9] provides a two-dimensional model and [6] a beam model, it can be concluded that no two-dimensional effect is acting in this case: the trapped transverse modes described in [9] thus do not seem to play any role.

\subsection{Comparisons and discussion}

The result of the model of [6] for the estimated parameters and the experimental results in the aluminium case are plotted on Fig. 10. The modulus of $R$ is of the same order of magnitude up to $4000 \mathrm{~Hz}$ and displays oscillations in the two cases, although it is more irregular in the experimental case. Above $4000 \mathrm{~Hz}$, the experimental reflection coefficient is slightly smaller in modulus. The phases are very similar in their order of magnitude.

The tapered extremities are usually far from perfect and may present irregularities [11]. Available models do not take into account these imper-

400 fections. They may allow the excitation of two-dimensional modes described in [9] by an incident wave and thus cause energy transfer and enhancement 

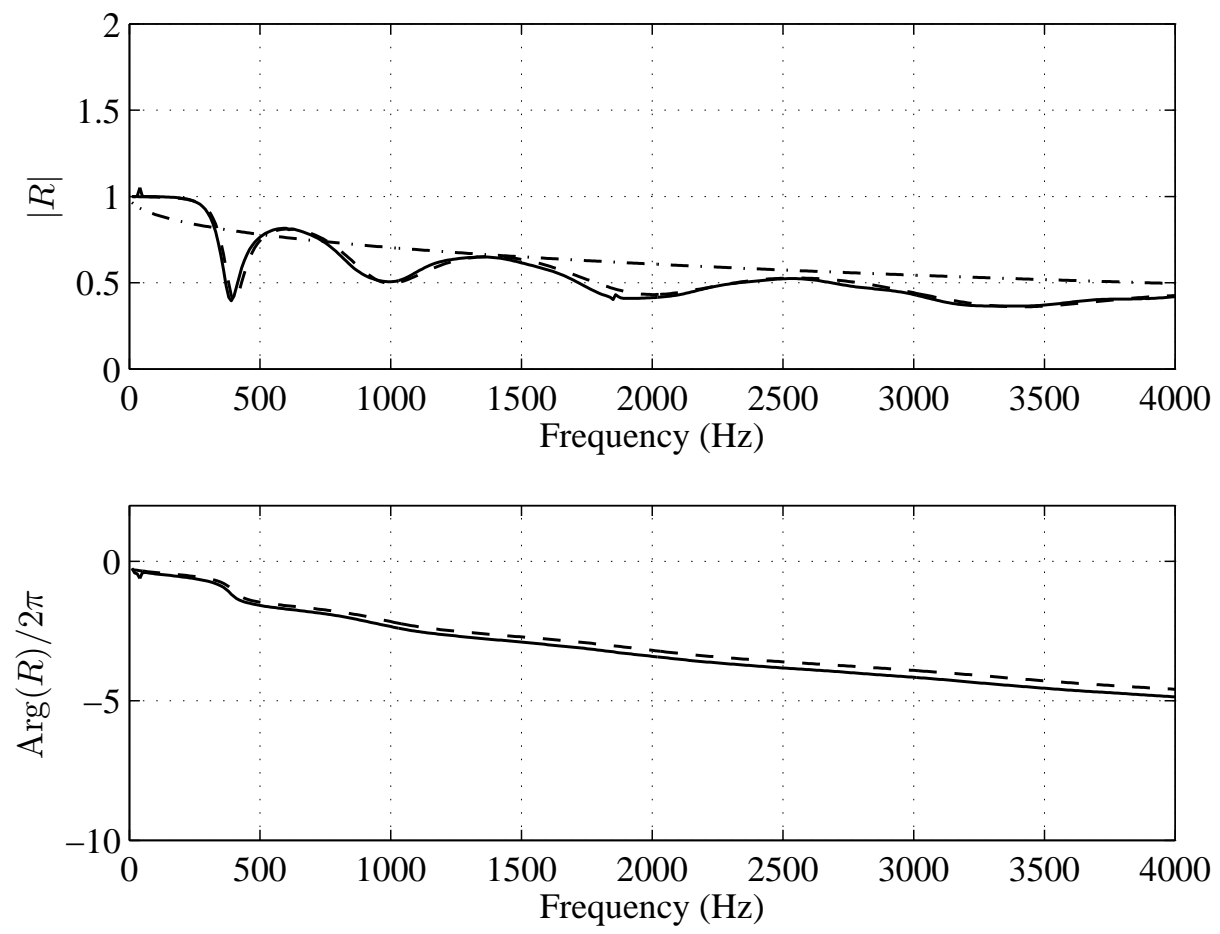

Figure 9: Reflection coefficient calculated with geometrical model (dash-dotted line), beam waveguide model (dashed line) and plate model (full line) in (a) modulus and (b) phase. 
of the ABH effect. Such a phenomenon can contribute to the differences between model and experiment.

(a)

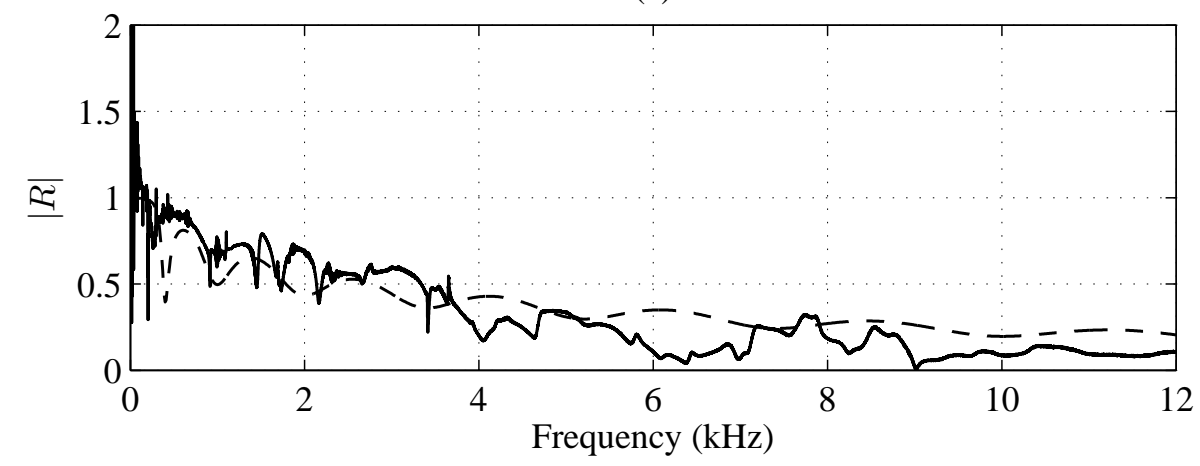

(b)

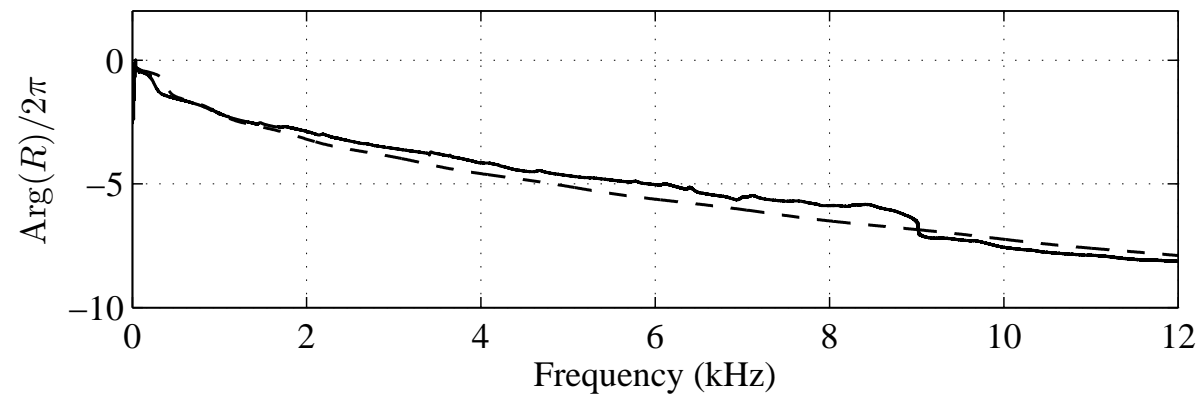

Figure 10: Reflection coefficient calculated with beam waveguide model (dashed line) and estimated experimental (full line) reflection coefficient in (a) modulus and (b) phase.

\section{Conclusions}

This paper is dedicated to the experimental measurement of the reflection coefficient of an Acoustic Black Hole termination. In Sec. 2, an experimental method for estimating the reflection coefficient of a beam extremity is presented and applied to beams with Acoustic Black Hole termination. In Sec. 3 

result for a free end, for two different materials. It is observed that the reflection coefficient of the $\mathrm{ABH}$ termination decreases with increased frequency and presents oscillations. The phase of the reflection coefficient rolls down, indicating a relatively important travel time in the tapered extremity. It to be quasi-constant with respect to frequency. More detailed study could be done to investigate in depth the links between geometry, material and correction length. A review of the existing models is made in Sec. 4; a comparison of the models between them shows that a beam model and plate model yield the same result but that a geometrical acoustics approach is not adapted to the studied geometry, which may not have a sufficient smoothness. A comparison between the model and the experiment shows a good agreement even if the experimental result is less regular than the result given by the model. It can be concluded that the estimation method proves to be usable and that the available models are satisfying to describe the overall behaviour of $R$. Besides, modelling the imperfections at the extremity may improve the adequacy to the experiment.

\section{Acknowledgements}

The authors are grateful to the Centre de Transfert de Technologies du Mans (CTTM) for the manufacture of the polymer beams used in this study and to Stanislas Renard for the manufacturing of the aluminium ABH terminations. 


\section{References}

${ }_{435}$ [1] D. Ross, E.L. Ungar, E.M. Kerwin, Damping of plate flexural vibrations by means of viscoelastic laminae, in: Structural damping, Pergamon Press Edition, J.E. Ruzicka, Oxford, 1960, pp. 49-57.

[2] A.D. Nashif, D.I.G. Jones, J.P. Henderson, Vibration Damping, A. Wiley-Interscience Publication, 1985.

[3] C. Vemula, A.N. Norris, G.D. Cody, Attenuation of waves in plates and bars using a graded impedance interface at edges, Journal of Sound and Vibration 196 (1) (1996) 107-127.

[4] V.V. Krylov, New type of vibration dampers utilising the effect of acoustic 'black holes', Acta Acustica United with Acustica 90 (2004) 830-837.

445

[5] V.V. Krylov, R.E.T.B. Winward, Experimental investigation of the acoustic black hole effect for flexural waves in tapered plates, Journal of Sound and Vibration 300 (1-2) (2007) 43-49.

[6] V.B. Georgiev, J. Cuenca, F. Gautier, L. Simon, V.V. Krylov, Damping of structural vibrations in beams and elliptical plates using the acoustic black hole effect, Journal of Sound and Vibration 330 (11) (2011) 24972508 .

[7] M.A. Mironov, Propagation of a flexural wave in a plate whose thickness decreases smoothly to zero in a finite interval, Soviet Physics: Acoustics 34 (3) (1988) 318-319. 
[8] V.B. Georgiev, J. Cuenca, F. Gautier, M. Moleron, L. Simon, Numerical and experimental investigation of the acoutic black hole effect for vibration damping in beams and elliptical plates, in: Euronoise 2009, Edinburgh, 2009.

[9] V. Denis, A. Pelat, F. Gautier, B. Elie, Modal overlap factor of a beam with an acoustic black hole termination, Journal of Sound and Vibration 333 (2014) 2475-2488.

[10] F. Gautier, J. Cuenca, V.V. Krylov, L. Simon, Experimental investigation of the acoustic black hole effect for vibration damping in elliptical plates., in: Acoustic'08, Paris, 2008.

[11] E.P. Bowyer, D.J. O’Boy, V.V. Krylov, J.L. Horner, Effect of geometrical and material imperfections on damping flexural vibrations in plates with attached wedges of power law profile, Applied Acoustics 73 (5) (2012) 514-523.

[12] E.P. Bowyer, D.J. O’Boy, V.V. Krylov, F. Gautier, Experimental inverstigations of damping flexural vibrations in plates containing tapered indentations of power-law profile, Applied Acoustics 74 (2013) 553-560.

[13] D.J. O'Boy, V.V. Krylov, Damping of flexural vibrations in circular plates with tapered central holes, Journal of Sound and Vibration 330 (10) (2011) 2220-2236.

[14] O. Aklouche, A. Pelat, S. Maugeais, F. Gautier, Scattering of flexural waves from an acoustic black hole in an infinite thin plate, in: MEDYNA 2013, Marrakech 23-25 april 2013, 2013. 
[15] O. Aklouche, A. Pelat, S. Maugeais, F. Gautier, Modle de la diffusion des ondes de flexion par un trou noir acoustique bi-dimensionnel, in: Congrs Franais d'Acoustique, Poitiers, 22-25 april 2014, 2014.

[16] Y.K. Lin, B.K. Donaldson, A brief survey of transfer matrix techniques with special reference to the analysis of aircraft panels, Journal of Sound and Vibration 10 (1) (1969) 103-143.

[17] B.R. Mace, Wave reflection and transmission in beams, Journal of Sound and Vibration 97 (1984) 237-246.

[18] J. Linjama, T. Lahti, Estimation of bending wave intensity in beams using the frequency response technique, Journal of Sound and Vibration 153 (1) (1992) 21-26.

[19] J. Linjama, T. Lahti, Measurement of bending wave reflection and impedance in a beam by the structural intensity technique, Journal of Sound and Vibration 161 (2) (1993) 317-331.

[20] X. Carniel, J.-C. Pascal, Vibrational power flow measurement: structure characterization and wave reflection coefficient measurement, in: Proceedings INTER-NOISE 85, Munich, 18-20 September 1985, 1985, pp. 613-616.

[21] J.-B. Piaud, J. Nicolas, La mesure de l'impdance mcanique, in: Proceedings 12th ICA, Toronto, 24-31 July 1986, Paper M2-7, 1986.

[22] F. Gautier, M.-H. Moulet, J.-C. Pascal, Reflection, transmission and coupling of longitudinal and flexural waves at beam junctions. part I: 
Measurement methods, Acta Acustica United with Acustica 92 (2006) 982-997.

[23] M.-H. Moulet, F. Gautier, Reflection, transmission and coupling of longitudinal and flexural waves at beam junctions. part ii: Experimental and theoretical results, Acta Acustica United with Acustica 93 (2007) $37-47$.

[24] K.P. Menard, Dynamic mechanical analysis: a practical introduction, CRC Press, 1999.

[25] J.D. Ferry, Viscoelastic properties of Polymers, New York: John Wiley, 1980.

[26] R. Penrose, A generalized inverse for matrices, Proceedings of the Cambridge Philosophical Society 51 (1955) 406-413.

[27] L.E. Kinsler, A.R. Frey, A.B. Coppens, J.V. Sanders, Fundamentals of acoustics, fourth edition, John Wiley \& Sons, 2000.

[28] M.A. Mironov, V.V. Pislyakov, One-dimensional acoustic waves in retarding structures with propagation velocity tending to zero, Acoustical Physics 48 (3) (2002) 400-405.

[29] V.V. Krylov, F.J.B.S. Tilman, Acoustic 'black holes' for flexural waves as effective vibration dampers, Journal of Sound and Vibration 274 (3-5) (2004) 605-619.

[30] A.N. Norris, Reflection and transmission of structural waves at an inter- 
face between doubly curved shells, Acta Acustica United with Acustica 84 (1998) 1066-1076.

[31] S. Felix, V. Pagneux, Multimodal analysis of acoustic propagation in three-dimensional bends, Wave Motion 36 (2) (2002) 157-168.

${ }_{525}$ [32] P.A. Feurtado, S.C. Conlon, F. Semperlotti, A normalized wave number variation parameter for acoustic black hole design, The Journal of the Acoustical Society of America 136 (2014) EL148. 